\title{
To Use or Not to Use Opioid Analgesia for Acute Abdominal Pain Before Definitive Surgical Diagnosis? A Systematic Review and Network Meta-Analysis
}

\author{
Paschalis Gavriilidis $^{\mathrm{a}, \mathrm{d}}$, Nicola de'Angelis ${ }^{\mathrm{b}}$, Aurelio Tobias ${ }^{\mathrm{c}}$
}

\begin{abstract}
Background: Despite the existing evidence, many physicians are reluctant to use opioid analgesia for acute abdominal pain.

Methods: We performed updated conventional and network meta-analyses. For the first time to our knowledge, direct and indirect evidence of any type of opioid analgesia was estimated and compared using network meta-analysis.

Results: There was no significant difference in the intensity of pain between the two cohorts (mean difference (MD) $=0.43(-0.05$ to $0.91), P=0.08)$. In addition, no significant difference was detected in the rate of incorrect diagnoses between the opioid analgesia and the placebo cohorts (odds ratio $(\mathrm{OR})=0.79(0.54$ to 1.17$), \mathrm{P}$ $=0.24$ ). Network meta-analysis demonstrated that the results of direct evidence of head-to-head comparisons of opioid analgesics with placebo were in accordance with the results of conventional metaanalysis. Moreover, estimation and comparison of the indirect evidence on the four opioid analgesics did not demonstrate significant differences in effect size.
\end{abstract}

Conclusions: Any type of opioid analgesic can be used safely for acute abdominal pain without risk of impairment of diagnostic accuracy.

Keywords: Opioid analgesia; Abdominal pain; Diagnosis; Morphine

\footnotetext{
Manuscript submitted November 11, 2018, accepted December 11, 2018

aDepartment of General and Colorectal Surgery, Northern Lincolnshire and Goole, Diana, Princess of Wales Hospital, Scartho Road, Grimsby DN33 2BA, UK

${ }^{b}$ Department of Digestive Surgery, Henri Mondor University Hospital, 94010 Creteil, France

'Tommy's National Centre for Miscarriage Research, Institute of Metabolism and Systems Research, University of Birmingham, Birmingham B15 2TG, UK ${ }^{\mathrm{d} C}$ Corresponding Author: Paschalis Gavriilidis, Department of General and Colorectal Surgery, Northern Lincolnshire and Goole, Diana, Princess of Wales Hospital, Scartho Road, Grimsby DN33 2BA, UK.

Email: pgavrielidis@yahoo.com
}

doi: https://doi.org/10.14740/jocmr3690

\section{Introduction}

Despite existing evidence that suggests its safety in patients with acute abdominal pain without the risk of obscuring the diagnosis [1], many physicians are still reluctant to use opioid analgesia in this situation [2].

We aimed to update existing evidence on the use of any type of opioid analgesia for acute abdominal pain using both conventional and network meta-analyses. Furthermore, for the first time to our knowledge, we evaluate direct and indirect evidence with different types of opioid analgesics and compare them using network meta-analysis. The primary outcome was the rate of incorrect diagnosis.

\section{Methods}

This systematic review and network meta-analysis were carried out in accordance with the guidelines set out in the Preferred Reporting Items for Systematic Reviews \& Meta-Analyses (PRISMA) checklist [3].

\section{Literature search}

A systematic literature search of articles published in the last 30 years was performed using the EMBASE, MEDLINE (PubMed), Cochrane Library, and Google Scholar databases using free text and Medical Subject Headings (MeSH) search terms (opioid analgesia or analgesics; acute abdominal pain or surgical pain; acute appendicitis; surgical diagnosis or definite diagnosis; morphine; randomized controlled trial). A grey literature search at the website clinicalTrials.gov was also performed. References of retrieved articles were checked manually for further relevant studies. Disagreement between authors was resolved by consensus.

\section{Study selection, inclusion and exclusion criteria}

We included randomized controlled trials (RCTs) that compared opioid analgesia of any type vs. placebo for acute surgical pain before definitive surgical diagnosis in patients older than 12 years, and if the mean age of subjects in the study 
sample was within the adult range. Non-randomized studies, reviews, and narrative articles were excluded.

\section{Data extraction and outcomes}

Two reviewers (PG and NA) independently extracted the following summary data from the included studies: name of authors; number of patients; age; type of opioid analgesia; pretreatment intensity of the pain; change in the intensity of the pain; rate of errors in treatment; and rate of incorrect diagnosis.

\section{Risk of bias assessment of included studies}

Two authors (PG, NA) independently assessed the risk of selection, performance, attrition, detection, and reporting bias for each included study. The above parameters were categorized as high, low, or unclear risk according to the Cochrane Handbook for Systematic Reviews of Interventions [4].

\section{Statistical analysis}

Statistical analysis was conducted using both Stata (version 15, Stata Corp LP, Collage Station, TX, USA) and Review Manager 5.3 software (The Cochrane Collaboration, Oxford, England). The $\mathrm{I}^{2}$ test was performed with cut-off values set at $25 \%, 50 \%$, and $75 \%$ to indicate low, moderate, and high levels of heterogeneity respectively [5]. In such cases, we performed analysis using both fixed- and random-effects models and the conclusions were compared; the random-effects model was used preferentially in case of discrepancies between the two models. In case of $\mathrm{I}^{2}$ values less than $25 \%$, the fixed-effect model was used throughout.

Dichotomous variables were analyzed based on odds ratios (OR) with 95\% confidence intervals (CIs). For the analyzed outcomes, the reference categories were selected to favour opioid analgesia if the OR was $<1$. Continuous variables were compared based on both the mean difference (MD) and the standardized mean difference (SMD). For studies that did not report the means and variances for the two groups, these values were estimated from the median, range, and the sample size where possible, using the technique described by Hozo et al [6]. For all analyses, the significance level was set at $\mathrm{P}<$ 0.05 .

Network meta-analysis was performed using hierarchical random-effects models [7]. A fixed-effects model was also used to estimate whether any discrepancy could be demonstrated between the results of the two models. Quantitative data synthesis of the connected network of studies was conducted using the software package WinBUGS (version 1.4.3, MRC Biostatistics Unit, Cambridge, UK) [8]. Pooled estimates were obtained using the Markov chain Monte Carlo method. Minimally informative priors with vague normal prior distributions were used. For each model, 200,000 simulations were generated for the two sets of different initial values, and the first 5,000 were discarded as the burn-in period. The Brooks-Gelman-

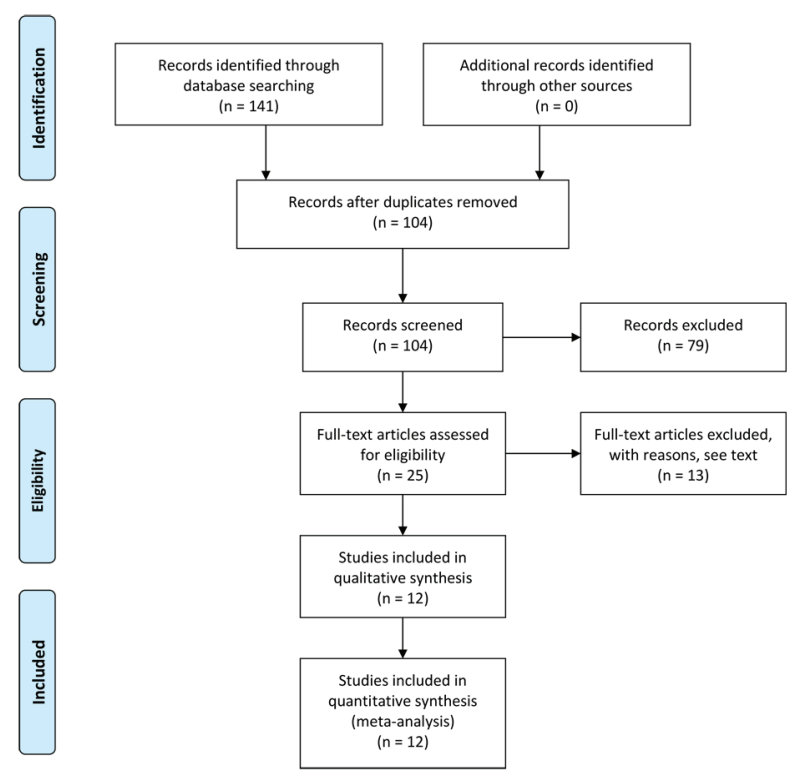

Figure 1. Flow diagram of the search strategy [3].

Rubin statistic was used for the assessment of convergence. The point estimate was defined as the median of the posterior distribution based on 200,000 simulations; the corresponding $95 \%$ credible intervals (CrIs) were obtained using the 2.5 th and 97.5 th percentiles of the posterior distribution, which can be interpreted in a similar way as $95 \%$ CIs. Inconsistency and heterogeneity of direct and indirect evidence for the five approaches were estimated.

\section{Sensitivity analysis}

Analyses of primary and secondary outcomes were conducted using both random- and fixed-effect models in order to assess the impact of heterogeneity on the results. Direct and indirect evidence of the five pain management approaches were estimated and compared. Publication bias was not estimated because less than 10 studies were included in each evaluated outcome [9].

\section{Results}

\section{Search strategy and study characteristics}

Twelve studies, which included 1,314 patients, were selected from a pool of 141 studies [10-21]. From the 25 full-text assessed studies, 13 were excluded for the following reasons: four studies used non-opioid analgesia, four included pediatric population, two were retrospective studies, two were case-controlled studies, and data extraction was difficult in one study (Fig. 1) [3]. Eight studies compared morphine to placebo, two 
Table 1. : Study Characteristics and Risk of Bias

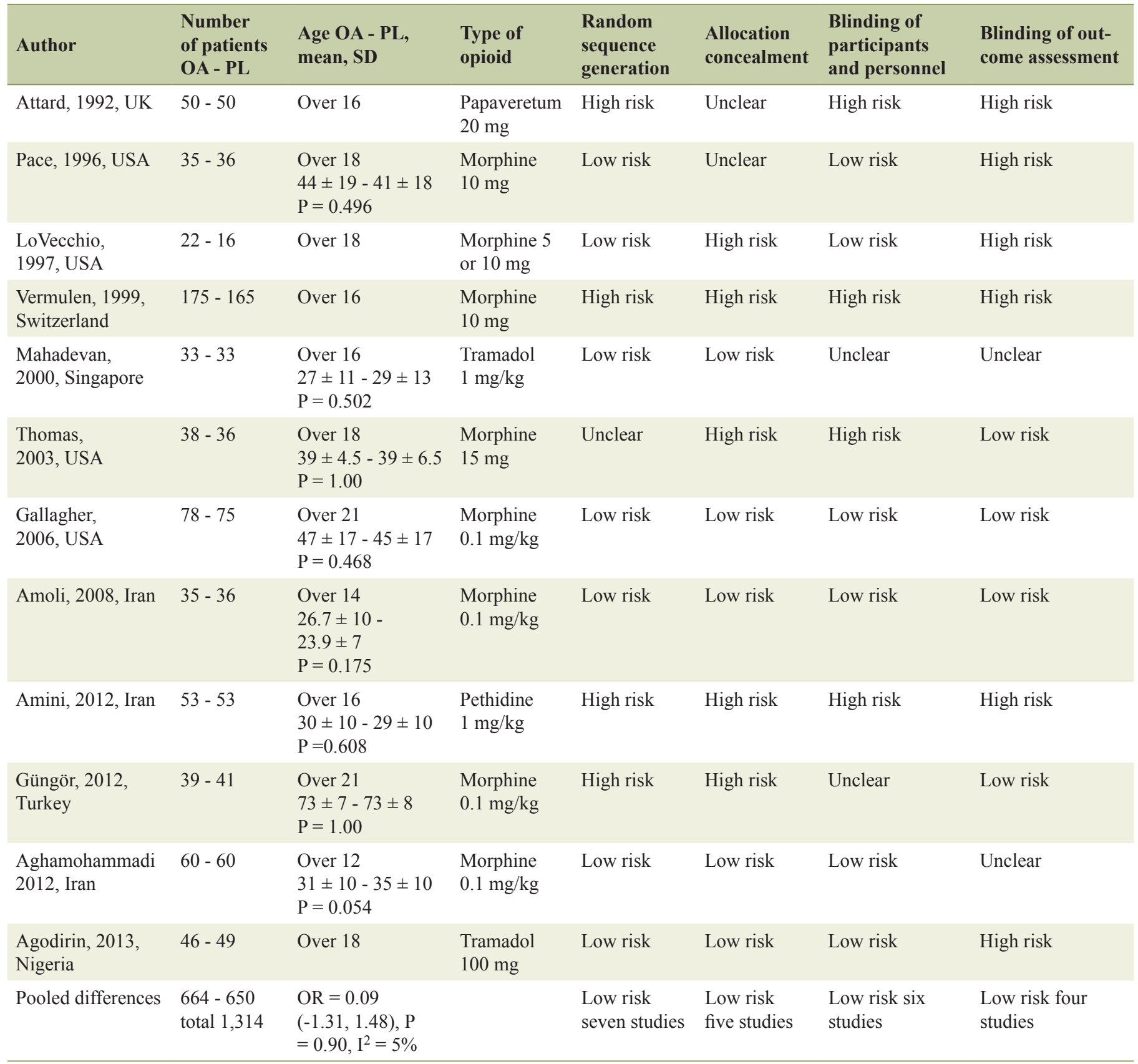

OA: opioid analgesia; PL: placebo; SD: standard deviation.

studies compared tramadol to placebo, one study compared papaveretum to placebo and one, pethidine to placebo (Table 1).

\section{Patient demographics}

There was no evidence of significant difference in age between the intervention and control cohorts. The mean age in each study was within the adult range, although some of the studies included patients over 12,14 , and 16 years old (Table 1 ).

\section{Results from pairwise meta-analysis of primary and sec- ondary outcomes}

\section{Incorrect diagnosis}

Nine of 12 studies reported incorrect diagnosis [10-13, 15-16, 18-19, 21]. There was no evidence of a statistically significant difference in the rate of incorrect diagnoses between the opioid analgesia cohort and the placebo cohort $(\mathrm{OR}=0.79(0.54$ to 


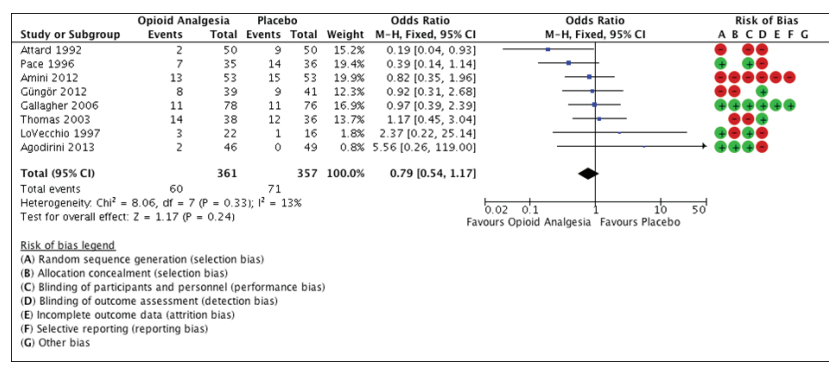

Figure 2. Forest plot illustrating incorrect diagnosis.

1.17), $\mathrm{P}=0.24, \mathrm{I}^{2}=13 \%$ ) (Fig. 2).

\section{Pre-treatment intensity of pain}

Studies reported pain using the visual analogue scale (VAS). Eleven out of 12 studies reported pre-treatment intensity of pain [10-20]. There was no evidence for statistically significant difference between the opioid analgesia cohort and the placebo cohort ( $\mathrm{MD}=0.43(-0.05$ to 0.91$\left.), \mathrm{P}=0.08, \mathrm{I}^{2}=81 \%\right)$.

\section{Post-treatment intensity of pain}

Reduction in pain was observed in both cohorts. However, reduction in the intensity of pain was significantly greater in the opioid analgesia cohort compared to the placebo cohort $(\mathrm{MD}=$ -1.76 ( -2.50 to -1.03$\left.), \mathrm{P}<0.001, \mathrm{I}^{2}=94 \%\right)$.

\section{Results from the network meta-analysis}

The network of evidence of the five pain management approaches was demonstrated using a figure of star with four radiuses. Vertices represent the pain management strategy and sample size. Lines represent head-to-head comparisons with line thickness being proportional to the number of studies included (Fig. 3a).

Direct evidence of incorrect diagnosis with any type of opioid analgesia vs. placebo

There was no evidence of statistically significant differences in incorrect diagnosis between the cohorts of morphine vs. placebo, tramadol vs. placebo and pethidine vs. placebo: OR $(\mathrm{CI})=0.96$ (0.64 to 1.44$), 5.56$ (0.26 to 119$)$, and 0.82 (0.35 to 1.96 ), respectively. Moreover, there was significantly fewer incorrect diagnosis with papaveretum compared to the placebo cohort $(\mathrm{OR}=0.19$ (0.04 to 0.93$))$ (Fig. 3b).

Indirect evidence of incorrect diagnosis with tramadol, papaveretum, and pethidine vs. morphine

There was no evidence of significant difference in incorrect

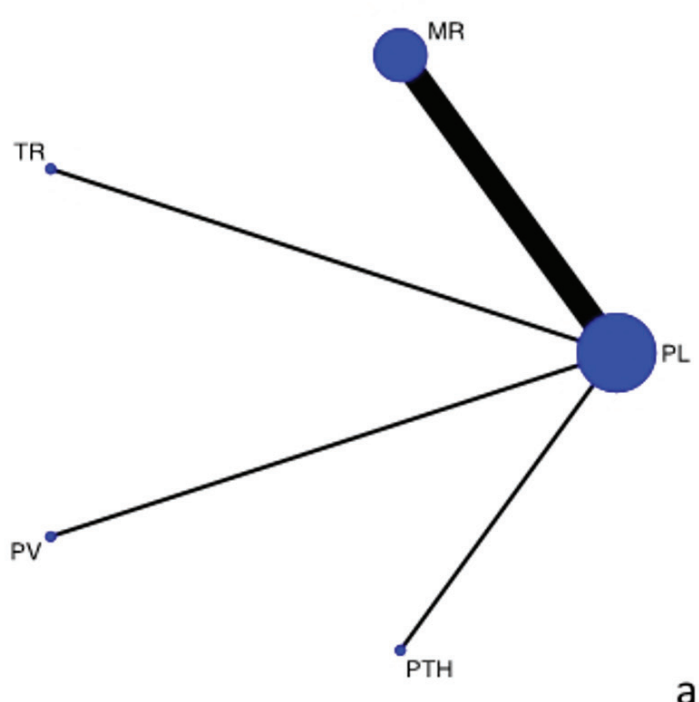

a

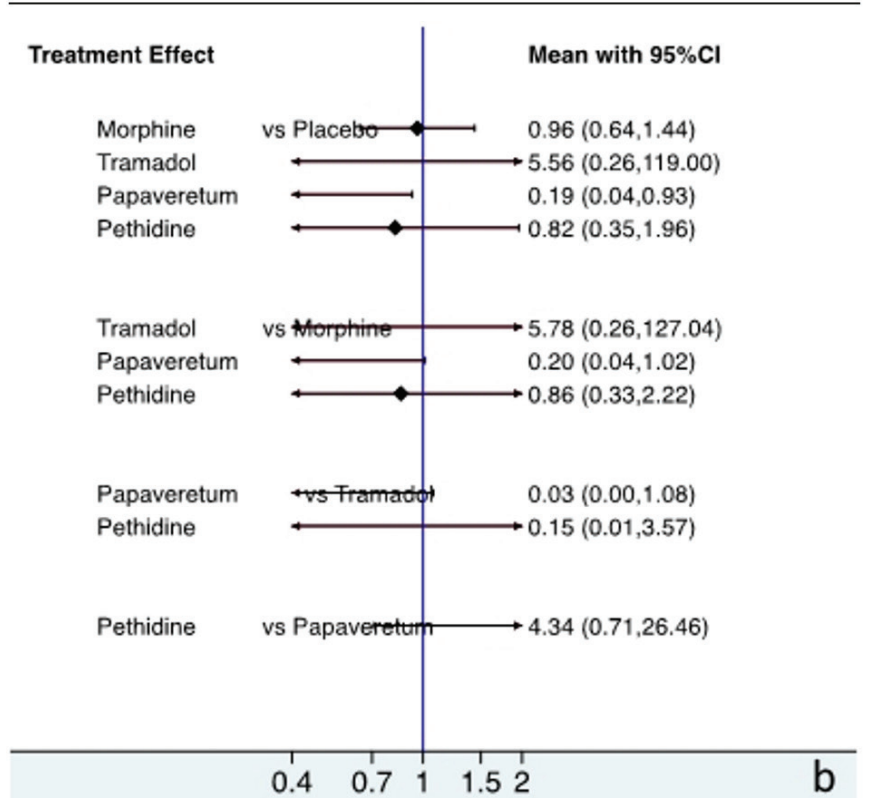

Figure 3. Plot showing (a) evidence of treatment approaches and (b) direct and indirect evidence of the treatment approaches. PL, placebo; MR, morphine; TR, tramadol; PV, papaveretum; PTH, pethidine.

diagnosis between the cohorts of tramadol vs. morphine, papaveretum vs. morphine, and pethidine vs. morphine: $\mathrm{OR}(\mathrm{CI})=$ 5.78 ( 0.26 to 127$), 0.20$ ( 0.04 to 1.01 ), and 0.86 ( 0.33 to 2.22 ), respectively (Fig. 3b).

Indirect evidence of incorrect diagnosis with papaveretum, and pethidine vs. tramadol

There was no evidence of significant difference in incorrect diagnosis between the cohorts of papaveretum and pethidine compared to tramadol: $\mathrm{OR}(\mathrm{CI})=0.03(0.00$ to 1.08$)$ and 0.15 (0.01 to 3.57 ), respectively (Fig. $3 b$ ). 
Indirect evidence of incorrect diagnosis with pethidine vs. papaveretum

There was no evidence of significant difference in incorrect diagnosis between the cohort of pethidine compared to papaveretum $(\mathrm{OR}=4.34$ (0.71 to 26.46)) (Fig. 3b).

\section{Risk of bias of RCTs}

Seven studies were assessed to be at low risk in random sequence generation, five were considered to be at low risk of allocation concealment, six were considered to be at low risk in the blinding of participants and personnel, and four studies were considered at low risk in the blinding of outcome assessment. Studies by Gallagher and Amoli were assessed to be at low risk in all the above domains (Table 1).

\section{Discussion}

The aim of this study was to estimate direct and indirect evidence of the impact of any type of opioid analgesia compared to placebo on acute abdominal pain before the establishment of definitive diagnosis using network meta-analysis.

There was no significant difference in the intensity of pain between the two cohorts, although a placebo effect was demonstrated. However, there was a significant reduction in the intensity of pain with opioid analgesics.

Despite pre-existing evidence, many clinicians are reluctant to use opioid analgesia in acute abdominal pain based on the belief that it impairs diagnostic accuracy $[1,2]$. The present study demonstrates that there is no significant difference in the rate of incorrect diagnosis between the opioid analgesic cohort and the placebo cohort.

Furthermore, in the present study direct and indirect evidence were estimated using network meta-analysis. The results of the direct evidence of network meta-analysis further justify the results of the conventional meta-analysis. Furthermore, for the first time to our knowledge, in the present study, all possible comparisons between four opioid analgesics were estimated based on indirect evidence provided by network meta-analysis. Consequently, the results demonstrated that there is no evidence of the superiority of one opioid analgesic over the other (Fig. $3 \mathrm{~b})$. Based on the results of the present indirect evidence, we can conclude that there is no need for further investigation in order to estimate the superiority of one opioid analgesic over the other, with all four opioids demonstrating a similar effect size.

To the best of our knowledge, this is the first network meta-analysis and the results of the present study further corroborate with the results of the previous Cochrane review [1]. Moreover, the present study is more representative because it includes studies from all continents (Table 1).

\section{Limitations}

However, the results of the present study should be interpreted cautiously, because it has several limitations. RCTs from the 1990s are not of good quality overall; none of them blinded the outcome assessors and their randomization and allocation concealment were inadequate. Another limitation of all the included studies is the small sample size, different clinical endpoints and variable lower age limits which contribute to study heterogeneity (Table 1). Therefore, an adequately powered, multicentre RCT with common objectives and a more homogenous population will shed further light on this topic.

\section{Conflict of Interest}

The authors declare no conflict of interest.

\section{Ethical Approval}

This study does not contain any studies with human participants or animals performed by any of the authors.

\section{Abbreviations}

MD: mean difference; PRISMA: Preferred Reporting Items for Systematic Reviews \& Meta-Analyses; OR: odds ratio; MeSH: Medical Subject Headings; RCTs: randomized controlled trials; SMD: standardized mean difference; CI: confidence interval; CrI: credible interval; VAS: visual analogue scale

\section{References}

1. Manterola C, Vial M, Moraga J, Astudillo P. Analgesia in patients with acute abdominal pain. Cochrane Database Syst Rev. 2011;1:CD005660.

2. Ayoade BA, Tade AO, Salami BA, Oladapo O. Administration of analgesics in patients with acute abdominal pain: a survey of the practice of doctors in a developing country. Int J Emerg Med. 2009;2(4):211-215.

3. Moher D, Liberati A, Tetzlaff J, Altman DG, The PRISMA Group. Preferred reporting items for systematic reviews and meta-analyses: the PRISMA statement. PLoS Med. 2009;6(7):e1000097.

4. Higgins JPT, Greens S. Cochrane handbook for systematic reviews of interventions version 5.1 [update March 2011]. The Cochrane Collaboration 2011. www.cochrane. handbook.org.

5. Higgins JP, Thompson SG, Deeks JJ, Altman DG. Measuring inconsistency in meta-analyses. BMJ. 2003;327(7414):557560.

6. Hozo SP, Djulbegovic B, Hozo I. Estimating the mean and variance from the median, range, and the size of a sample. BMC Med Res Methodol. 2005;5:13.

7. Lu G, Ades AE. Combination of direct and indirect evidence in mixed treatment comparisons. Stat Med. 2004;23(20):3105-3124.

8. Ades AE, Sculpher M, Sutton A, Abrams K, Cooper N, 
Welton N, Lu G. Bayesian methods for evidence synthesis in cost-effectiveness analysis. Pharmacoeconomics. 2006;24(1):1-19.

9. Harbord RM, Harris RJ, Sterne JA. Updated tests for small-study effects in meta-analyses. The Stata Journal. 2009;9:197-210.

10. Attard AR, Corlett MJ, Kidner NJ, Leslie AP, Fraser IA. Safety of early pain relief for acute abdominal pain. BMJ. 1992;305(6853):554-556.

11. Pace $\mathrm{S}$, Burke TF. Intravenous morphine for early pain relief in patients with acute abdominal pain. Acad Emerg Med. 1996;3(12):1086-1092.

12. LoVecchio F, Oster N, Sturmann K, Nelson LS, Flashner $\mathrm{S}$, Finger R. The use of analgesics in patients with acute abdominal pain. J Emerg Med. 1997;15(6):775-779.

13. Vermeulen B, Morabia A, Unger PF, Goehring C, Grangier C, Skljarov I, Terrier F. Acute appendicitis: influence of early pain relief on the accuracy of clinical and US findings in the decision to operate - a randomized trial. Radiology. 1999;210(3):639-643.

14. Mahadevan M, Graff L. Prospective randomized study of analgesic use for ED patients with right lower quadrant abdominal pain. Am J Emerg Med. 2000;18(7):753756.

15. Thomas SH, Silen W, Cheema F, Reisner A, Aman S, Goldstein JN, Kumar AM, et al. Effects of morphine analgesia on diagnostic accuracy in Emergency Department patients with abdominal pain: a prospective, randomized trial. J Am Coll Surg. 2003;196(1):18-31.

16. Gallagher EJ, Esses D, Lee C, Lahn M, Bijur PE. Randomized clinical trial of morphine in acute abdominal pain. Ann Emerg Med. 2006;48(2):150-160, e151-154.

17. Amoli HA, Golozar A, Keshavarzi S, Tavakoli H, Yaghoobi A. Morphine analgesia in patients with acute appendicitis: a randomised double-blind clinical trial. Emerg Med J. 2008;25(9):586-589.

18. Amini M, Alizade SH, Cyrus A, Khodam S, Rafeeie M, Hasanpur A, Solhi H. Does pethidine hydrochloride analgesia in patients with acute appendicitis alter the diagnostic accuracy of clinical evaluation: a randomized doubleblind clinical trial. Int J Medical Toxicology and Forensic Medicine. 2012;2:56-60.

19. Gungor F, Kartal M, Bektas F, Soyuncu S, Yigit O, Mesci A. Randomized controlled trial of morphine in elderly patients with acute abdominal pain. Ulus Travma Acil Cerrahi Derg. 2012;18(5):397-404.

20. Aghamohammadi D, Gholipouri C, Hosseinzadeh H, Khajehee MA, Ghabili K, Golzari SE. An evaluation of the effect of morphine on abdominal pain and peritoneal irritation signs in patients with acute surgical abdomen. J Cardiovasc Thorac Res. 2012;4(2):45-48.

21. Agodirin O, Oguntola A, Adeoti M, Agbakwuru A, Oluwadiya K, Olofinbiyi B. Preoperative pain treatment in acute abdomen in Osogbo, Nigeria: a randomized double-blind placebo-controlled study. Int J Emerg Med. 2013;6(1):3. 